\title{
Postwachstum und Ressourcen- schonung
}

Eine sich vorrangig auf technologische Innovationen und Entlastungsgewinne stützende Wirtschafts- und Umweltpolitik wird für die dauerhafte Senkung der Ressourcennutzung nicht ausreichen. Es bedarf auch kultureller Veränderungen im Umgang mit Ressourcen und ambitionierter und wegweisender politischer Maßnahmen. Von Bettina Bahn-Walkowiak und Henning Wilts

\section{Einführung}

Vor dem Hintergrund der Frage, wie Postwachstumsgesellschaften eigentlich gestaltet werden könnten, wurden im Rahmen des vom Umweltbundesamt (UBA) geförderten Projekts Postwachstum und Ressourcenschonung, in dem unter anderem eine umfangreiche Analyse der Diskursströmungen vorgenommen wurde [1], eine Reihe von zentralen und häufig vorgeschlagenen politischen Instrumenten aus der Postwachstumsliteratur zu ihrem Ressourcenschonungspotenzial untersucht.

Aus der Kernliteratur der Degrowth-, Postwachstums- und Green-Growth-Diskurse waren dazu insgesamt über 40 unterscheidbare, wenngleich teilweise thematisch gleich gelagerte Instrumente und Maßnahmen identifiziert worden, von denen angenommen wurde, dass sie das Potenzial besitzen, den gesellschaftlichen Ressourcenverbrauch merklich zu verringern. [2]. Es zeigte sich indes schnell, dass die überwiegende Zahl von Vorschlägen kaum oder nicht einmal in Ansätzen empirisch untersucht ist.

Zehn ausgewählte Kerninstrumente (Abbildung 1) wurden entlang eines einheitlichen Analyserasters mit insgesamt zwölf Kategorien untersucht (Instrumententyp, Wirkungsmechanismus, betroffene Ressourcen, potenzielle oder empirische Ressourceneffekte, positive und negative Nebeneffekte, Problemverlagerungen, Verteilungsfragen, Umsetzungshemmnisse, Umsetzungsbeispiele sowie Forschungsbedarf).

Der Mangel an quantitativen empirischen Untersuchungen zu den Um- weltentlastungspotenzialen zentraler Vorschläge führte zu der pragmatischen, aber für einen ersten Einblick als hinreichend eingestuften qualitativen Unterscheidung zwischen „außerordentlich positiven“, „sehr positiven“, „positiven“ und „gering positiven Effekten“ sowie zur Kategorie „Wirkung nicht abschätzbar".

Auf dieser Basis und einer weitergehenden Betrachtung von Reboundeffekten, sozialen und ökonomischen Nebeneffekten und Problemverlagerungen wurde eine qualitative Priorisierung der Instrumentenvorschläge vorgenommen und zwischen hoher, mittlerer und niedriger Priorität auf einem potenziellen Postwachstumspfad unterschieden [3].

\section{Ressourcenschonungspotenziale}

\subsection{Außerordentlich positive Effekte}

Modellierungsergebnissen zufolge induzieren insbesondere ökologische Steuer zur Internalisierung externer Kosten deutliche Senkungen bei Rohstoff- und Energieverbrauch und $\mathrm{CO}_{2}$-Emissionen durch Nutzung von Preismechanismus und Nachfragelenkung im Markt (Meyer et al. 2018). In Abhängigkeit von Umsetzungsbreite, -tiefe und Steuerhöhe ist der Beitrag zu den unterschiedlichen Ressourcen divergent und der Beitrag zu Biodiversität und Flächenverbrauchssenkungen indirekt und abhängig von den durch eine Steuerreform unmittelbar adressierten Sektoren sowie entstehenden Substitutionsund Reboundeffekten (Santarius 2015).

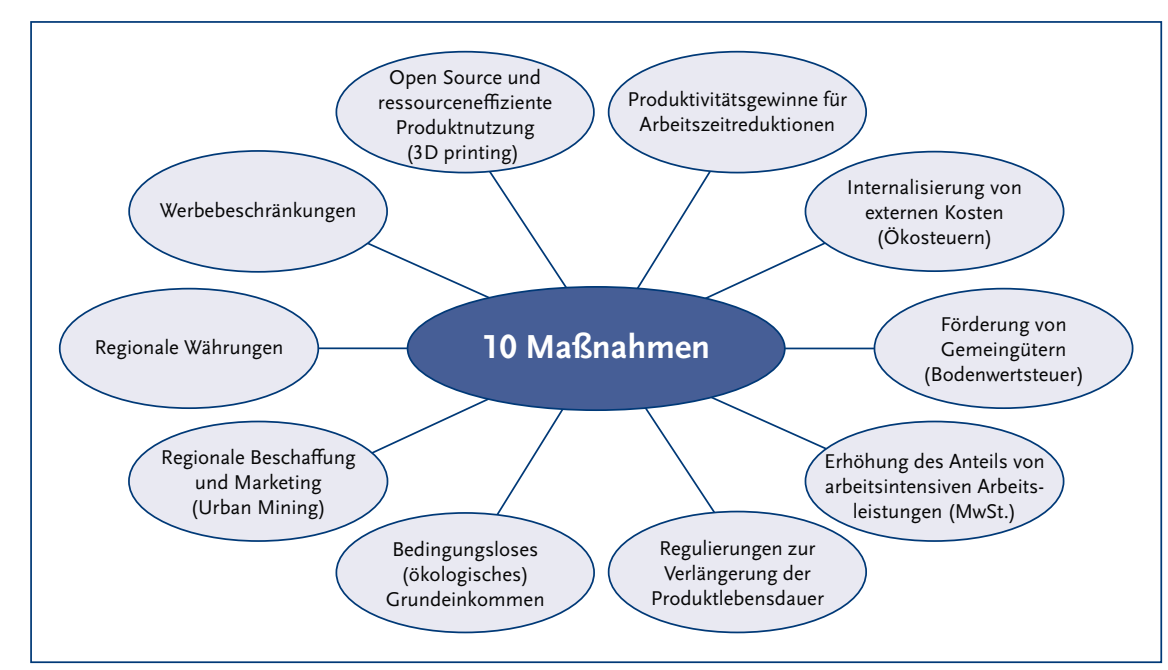

Abbildung 1: Auswahl von zehn Instrumenten aus der Postwachstumsdiskussion für die Detailanalyse permits copying and redistributing the material in any medium or format, provided the original work is properly cited, it is not used for commercial purposes and it is not remixed, transformed or built upon. The access to the digital version of this article is reserved to subscribers of ÖkologischesWirtschaften until two years after the date of publication; after two years it is available to all readers. 


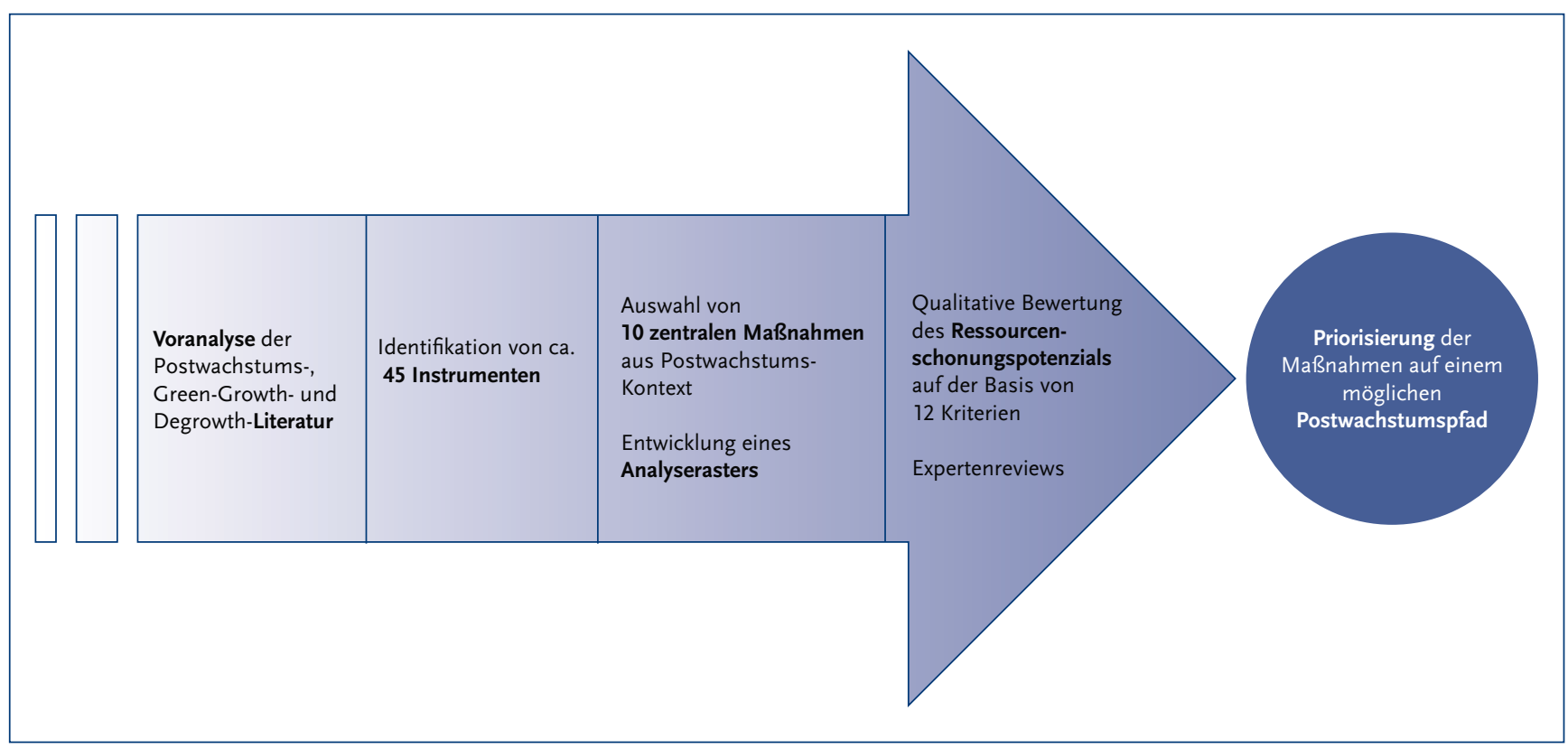

Abbildung 2: Methodisches Vorgehen

Obwohl der positive Wirkungsmechanismus von Ökosteuern hinlänglich bekannt ist, befindet sich Deutschland im EU-Vergleich inzwischen im unteren Drittel der Umweltbesteuerung und die Wirkung des geltenden Systems ist weitgehend verpufft (UBA 2018). Durch Erhöhung bereits bestehender Steuern auf umweltbelastende Aktivitäten oder neu einzuführende Abgaben können stärkere wirtschaftliche Anreize gesetzt (Bachus et al. 2017) sowie eine Entlastung arbeitsbezogener Steuern durch alternative Verwendung des Aufkommens vorgenommen werden (Ludewig 2017). Umsetzungs- und Ausgestaltungsbeispiele finden sich neben Deutschland in Dänemark, Finnland, Niederlande, Schweden und in Großbritannien.

\subsection{Sehr positive Effekte}

Sehr positive regionale Wertschöpfungs- und Ressourcenschonungseffekte sind von Maßnahmen der regionalen Beschaffung und Vermarktung beziehungsweise der Stärkung der lokalen oder regional dezentralisierten Produktion zu erwarten (Cooke 1997; Schiller et al. 2010).

Der Beitrag zur Schonung natürlicher Ressourcen von regionaler Beschaffung und Vermarktung (im Projekt am Beispiel Baumineralien im Kontext Urban Mining beispielhaft untersucht) ist durch Verkürzung der Wertschöpfungsketten erheblich. Dabei entspricht das Ressourcenschonungspotenzial dem Volumen an Primärmaterial, auf dessen Einsatz bei der Betonerzeugung für den Hochbau aufgrund einer Substitution durch Recyclingbaustoffe verzichtet werden kann. Dieses muss aber mit Blick auf den Nettoeffekt mit dem Energieaufwand für Recyclingverfahren und Transport verrechnet werden. Der Beitrag zur Ressourcenschonung fällt dann besonders hoch aus, wenn energieeffiziente Verfahren - am besten auf Basis erneuerbarer
Energien - beim Recycling eingesetzt und eine Engführung der Stoffkreisläufe bei Baumineralien vorgegeben werden. Umsetzungsbeispiel ist hier die Stadt Zürich (Stadt Zürich 2009).

\subsection{Positive Effekte}

Grundidee der Verkürzung der (jährlichen, wöchentlichen oder Lebens-)Arbeitszeit im Rahmen von Postwachstumsmaßnahmen ist es, Produktivitätsgewinne nicht in Einkommensgewinne zu übersetzen, wie es zumeist in marktwirtschaftlichen Systemen gemacht wird, sondern als (Frei-)Zeitgewinne der Gesellschaft zur Verfügung zu stellen, um so mehr Spielraum für (potenziell) immaterielle Tätigkeiten (wie Ehrenämter) zu gewinnen. Seit den 1980er Jahren kann ein relativ konstantes Verhältnis von Arbeits- und Freizeit festgestellt werden, während sich das Einkommen relativ zu einem konstanten Zeitbudget stetig erhöht. Zeit gewinnt so bei steigendem materiellem Wohlstand an Wert, denn sie kann im Gegensatz zu Geld nicht akkumuliert werden! Infolgedessen ist der entgangene Nutzen durch fehlende Zeit größer als der entgangene Nutzen durch fehlendes Geld (Buhl et al. 2016). Als Praxisbeispiel können die Stadt Göteborg (allerdings mit vollem Lohnausgleich) sowie ein Modellprojekt der Firma Volkswagen benannt werden.

Diese positiven Reduktionspotenziale beruhen auf Studien, die eine direkte Beziehung zwischen Arbeitszeitreduktion und Umweltbelastung quantifizieren (z. B. Knight et al. 2013). Sie ermitteln die Senkung des inländischen Materialinputs, die Verkleinerung des ökologischen Fußabdrucks und die Verringerung der Treibhausgase, trotz Zeit-Rebound-Effekten, die ebenfalls entstehen können. Dazu kommen Nebeneffekte in Form von individueller Lebenszufriedenheit sowie ehrenamtlichem Engagement und stärkerer sozialer Gemeinschaftsbildung. 
Die allgemeine Ressourceninanspruchnahme sowie die $\mathrm{CO}_{2}$-Emissionen lassen sich in vielen Fällen auch durch verlängerte Produktnutzungsdauern deutlich reduzieren. Gegen ein frühes oder künstlich herbeigeführtes Ende der Lebensdauer von Produkten können Regelungen zur Produktlebensdauer wie einer Herstellergarantieaussagepflicht (Aussagen über die garantierte Lebensdauer von Produkten) (Broehl-Kerner et al. 2012; Maitra-Ekern et al. 2016; UBA 2016) eingeführt werden mit dem Ziel, damit veränderte Nutzungsstrukturen, längere Nutzungen und Wiederverwendung von Produkten zu befördern. In diesem Bereich kann derzeit Frankreich als Praxisbeispiel benannt werden. Verpflichtende Angaben zur absehbaren Lebensdauer eines Produkts in Kombination mit Aussagen zur Ressourceninanspruchnahme für Produktion und Betrieb könnten Herstellungsverfahren beeinflussen und ressourcenbewussteren Konsum ermöglichen.

Open-Source-Ansätze haben in den vergangenen Jahren deutlich an Bedeutung gewonnen und als internetbasierte Ansätze eine Vielzahl von neuen Möglichkeiten eröffnet, Informationen auch zu Produkten global zu teilen und gemeinsam weiterzuentwickeln (Townsend et al. 2011). Eine verpflichtende Offenlegung von relevanten Produktinformationen durch die Hersteller zum Beispiel für Produkte, die in Deutschland oder auf dem europäischen Binnenmarkt angeboten werden, wäre denkbar, um die Herstellung und Verfügbarkeit von Reparaturund Ersatzteilen zu verbessern. In diesem Sinne könnten im Rahmen von Ressourcenschutz-Strategien die bisher freiwilligen Ansätze zu Open-Source-Konzepten deutlicher unterstützt werden. Vorliegende Analysen verdeutlichen das technische Potenzial zum Beispiel des 3-D-Drucks und die damit verbundenen Ressourceneinsparpotenziale durch Verringerung oder Vermeidung von Abfall, die Ermöglichung komplexer Leichtbaustrukturen und die Aufwandsminderung für Transport und Logistik trotz energetischer Mehraufwände bei der Produktion.

Die fortschreitende Digitalisierung wird erkennbar massive Einflüsse auf das Design von Produkten und damit auch auf Produktions- und Konsummuster haben (Petschow et al. 2014). Das Thema Open Sources und transparente Produkteigenschaften könnten dabei ein Ansatz sein, die Möglichkeiten der Digitalisierung auch zur Senkung des Ressourcenverbrauchs zu nutzen.

\subsection{Geringe positive Effekte}

Ein ermäßigter Mehrwertsteuersatz auf bestimmte arbeitsintensive Dienstleistungen - zum Beispiel für Reparaturdienstleistungen - würde diese Tätigkeitsbereiche steuerlich begünstigen mit dem Ziel, den Leistungsanbietern Preissenkungen zu ermöglichen, die damit Anreize für den Endabnehmer setzen können. Damit kann die Nachfrage nach dieser Dienstleistung am Markt gestärkt werden. Durch Wieder- und Weiterverwendung von Produkten (wie Schuhe, Textilien) in Haushalten, die unter anderen Umständen der Entsorgung zugeführt werden, werden Beiträge zur Ressourcenschonung geleistet (Poppe 2014). Zudem können neue Arbeitsplätze geschaffen, Schwarzarbeit beziehungsweise Schattenwirtschaft eingedämmt (Kornhardt 2009) und Abfallvermeidung sowie Maßnahmen zur energetischen Sanierung befördert werden. Der Effekt ist von der durch die Preissenkungen erzielten Nachfragewirkung abhängig, denn die Dynamik der Preisüberwälzung unterscheidet sich in Teilmärkten aufgrund der variierenden Wettbewerbsintensität teilweise erheblich. Ermäßigte Mehrwertsteuersätze für arbeitsintensive Dienstleistungen finden sich in Griechenland, Irland, Niederlande, Luxemburg, Polen, Slowenien, Finnland und Schweden.

Aktuelle Diskussionen über eine Bodenwertsteuer beziehen sich in der Regel auf eine Umwandlung der Grundsteuer in eine Bodenwertsteuer. Als mögliches Substitut einer Grundsteuer unterscheidet die Bodenwertsteuer zwischen Boden und Gebäudebestand, da der Wert des Bodens durch die Erschließungs- und Infrastrukturleistungen des öffentlichen Sektors geprägt ist, während der Wert der Gebäude von den Investitionen des jeweiligen Eigentümers abhängt (Dye et al. 2015). Auf Grundlage der theoretischen Erkenntnisse sind damit positive Wirkungen in Bezug auf Ressourcenschonung zu erwarten, wie zum Beispiel stärkere Anreize für Gebäudeinstandhaltungen und energetische Sanierungen sowie ein Rückgang des spekulativen Hortens unbebauter Grundstücke. Die Auswirkungen auf die Zersiedlung als Problem mit dem größten Potenzial zur Ressourcenschonung wurden bisher nur theoretisch und mit kontroversen Ergebnissen untersucht. Unterschiedliche Formen einer Bodenwertsteuer finden sich in den USA, Australien, Neuseeland, Kenia, Südafrika und Estland.

\begin{tabular}{|c|c|c|c|c|c|}
\hline $\begin{array}{ll}\text { Priorität } & \text { Effekte } \\
\end{array}$ & $\begin{array}{l}\text { außerordentlich } \\
\text { positiv }\end{array}$ & sehr positiv & positiv & gering positiv & nicht abschätzbar \\
\hline $\begin{array}{l}\text { hoch } \\
\text { (auch als Einzelinstrumente } \\
\text { empfehlenswert) }\end{array}$ & $\begin{array}{l}\text { Ökosteuern } \\
\text { (Internalisierung } \\
\text { externer Kosten) }\end{array}$ & Regionales Wirtschaften & $\begin{array}{l}\text { Open-Source-Ansätze } \\
\text { (transparente Produkt- } \\
\text { eigenschaften) }\end{array}$ & & \\
\hline $\begin{array}{l}\text { mittel } \\
\text { (als Element eines Politik- } \\
\text { mixes) }\end{array}$ & & & $\begin{array}{l}\text { Arbeitszeitreduktionen } \\
\text { Verlängerung Produkt- } \\
\text { lebensdauer }\end{array}$ & $\begin{array}{l}\text { ermäßigte MwSt.-Sätze } \\
\text { auf Dienstleistungen } \\
\text { Werbebeschränkungen }\end{array}$ & \\
\hline $\begin{array}{l}\text { niedrig } \\
\text { (ggf. ergänzendes Instrument/ } \\
\text { Forschungsbedarf) }\end{array}$ & & & & Bodenwertsteuer & $\begin{array}{l}\text { Regionalwährungen } \\
\text { ökologisches } \\
\text { Grundeinkommen }\end{array}$ \\
\hline
\end{tabular}

Tabelle 1: Einordnung der zehn analysierten Instrumente hinsichtlich ihrer Ressourcenschonungseffekte und Priorisierung für einen potenziellen Postwachstumspfad (Anmerkung: Qualitative Einschätzung anhand teils empirischer, teils explorativer Literatur) 


\section{„Die Effekte einer gestärkten Regional- wirtschaft für Einkommen, Beschäftigung und kommunale Haushalte sowie die Auswirkungen auf das Innovations- verhalten sind aus heutiger Sicht noch unklar."}

Angesichts der verschiedenen potenziell positiven Aspekte kann der Wirkungsmechanismus der Bodenwertsteuer bei einer möglichen Reform der Grundsteuer mitbedacht werden.

Werbebeschränkungen oder -verbote können als ordnungsrechtliches Instrument für verschiedene Produktgruppen vor dem Hintergrund der durch sie verursachten externen Umweltkosten verwendet werden. Ihre Begründung basiert zum Beispiel auf den Gesundheitsfolgekosten, die durch übermäßigen Konsum entstehen, wie etwa im Bereich des Fast Food, mit dem Ziel, vor allem Kinder in verschiedenen Medien (TV, Internet) zu schützen (Kerkhof et al. 2015). Eine analoge Verwendung wäre möglich für Produktgruppen mit hohem Ressourcenverbrauch und einem hohen Problemverschiebungscharakter auf globaler Ebene und für nachfolgende Generationen (zum Beispiel Flugreisen, Kreuzfahrten, Autos, die bestimmte $\mathrm{CO}_{2}$-Grenzwerte nicht einhalten), um so die Nachfrage oder zumindest die Zuwächse zu dämpfen und Entlastungen der $\mathrm{CO}_{2}$-Emissionen und des Ressourcenverbrauchs zu erreichen. Unterschiedliche Formen von Werbebeschränkungen finden sich derzeit in Schweden, Norwegen, Österreich, Flandern, Luxemburg, Griechenland, Italien, UK, Brasilien.

Werbung bewirbt und bewirkt Wachstum (DIW Econ 2016), wenngleich es dabei häufig um Marktanteile im sektoralen Wettbewerb geht. Das Ressourcenschonungspotenzial dieses Instruments ist zwar abhängig von den ausgelösten Nachfrageeffekten in den unterschiedlichen Produktgruppen und die Wirkung damit indirekt. Dass aber nachhaltige Nachfrageeffekte möglich sind, ist für den Bereich des Tabakkonsums belegt (Henriksen 2012).

\subsection{Wirkung nicht abschätzbar}

Als weitere, in ihrer Wirkung jedoch derzeit nicht einschätzbare Instrumente werden auch Regionalwährungen und ein ökologisches Grundeinkommen häufiger als zentrale Elemente einer Postwachstumsgesellschaft benannt.

Dabei gilt die Regionalwährung als ein Konzept, das mit einem nachhaltigeren und ressourcenbewussteren Lebensstil und verändertem Nachfrageverhalten wie Reduktion des Konsums, einem ausgeprägteren Reparaturverhalten, Wiederverwendung, Energieeinsparungen und Recycling assoziiert oder qualitativ abgeleitet wird. Es liegen jedoch keine quantifizierenden Analysen vor (Place et al. 2015).
Der Wirkungsmechanismus des ökologischen Grundeinkommens beruht auf zwei Säulen: Der Verteuerung von Ressourcenverbräuchen und Umweltbelastungen sowie der Umverteilung des durch die steuerliche Belastung gewonnenen Finanzaufkommens in einkommensschwächere Schichten (Haywood 2014; Schachtschneider 2014). Ein Umsetzungsbeispiel lag zur Zeit der Analyse nicht vor. Es bestehen daher Unsicherheiten hinsichtlich der Ressourceneffekte durch das Verhalten der Individuen (Arbeitsmarkt, Konsum) und der Unternehmen (Investitionen, Standortwahl) sowie am Arbeitsmarkt (Arbeitszeitreduktion/Ausbau der Care-Economy vs. mögliche Beschäftigungseffekte).

\section{Priorisierung der Vorschläge für einen Postwachstumspfad}

Die Analyse kommt zusammenfassend zu dem Ergebnis, dass es Ressourcenschonungspotenziale bei fast allen Maßnahmen gibt, die in einigen Fällen als weitgehend richtungssicher gelten können (Ökosteuern, Stärkung der Regionalwirtschaft, Verlängerung der Produktlebensdauer, Arbeitszeitverkürzung, transparente Produkteigenschaften). Auf Basis der vorgenommenen Analysen und weiterer Effekte (wie Reboundeffekte, soziale Nebenwirkungen, Problemverlagerungen) wurde eine abschließende qualitative Priorisierung mit Blick auf die Rolle, die diese Vorschläge auf einem Postwachstumspfad einnehmen könnten, vorgenommen.

Eine hohe Priorität werden der marktbasierten Internalisierung von bisher externalisierten Kosten des Ressourcenverbrauchs und der Umweltbelastungen, der Stärkung von regionaler Beschaffung und Vermarktung sowie der Stärkung von sogenannten Open Sources und den damit verbundenen transparenten Produkteigenschaften eingeräumt, um ressourceneffizienteren Konsum sehr viel deutlicher zu unterstützen. Diese Maßnahmen sollten auch als Einzelinstrumente umgesetzt werden.

Den Regelungen zur Verlängerung der Lebensdauer von Produkten, der Reduktion von Arbeitszeit, einer reduzierten Mehrwertsteuer auf arbeitsintensive Dienstleistungen (inklusive der systematischen Integration mit einer Öko-Steuerreform) und Werbebeschränkungen für bestimmte ressourcenintensive Produktgruppen wird eine mittlere Priorität auf einem Postwachstumspfad zugeordnet. Diese Instrumente sollten als wichtige Bausteine eines Politikmixes umgesetzt beziehungsweise in ihren für einen tatsächlichen Beitrag zum nachhaltigen Postwachstum notwendigen Rahmenbedingungen weiter präzisiert werden.

Schließlich wird der Bodenwertsteuer (in Ergänzung anderer fiskalischer Maßnahmen), dem ökologischen Grundeinkommen (ggf. im Kontext einer Öko-Steuerreform) und den Regionalwährungen (im Kontext der Stärkung dezentralen Wirtschaftens) eine eher niedrige Priorität zugeordnet, da sie aus heutiger Sicht zwar mittelfristig sinnvolle Maßnahmen beziehungsweise Ergänzungen der anderen Instrumente darstellen, aber gleich- 
zeitig den noch deutlichsten Forschungsbedarf aufweisen, um tatsächlich erfolgreich umgesetzt werden zu können.

Die umfassende Umgestaltung des Steuersystems im Hinblick auf eine stärkere Internalisierung von externalisierten Kosten von Ressourcenverbrauch sowie die Nachfragelenkung in Richtung eines ressourceneffizienteren Konsums muss als grundsätzlich erstrebenswert, ja geradezu unvermeidbar angesehen werden.

\section{Umsetzbarkeit, Zielkonflikte und Forschungsbedarf}

Die Untersuchung hat Konzepte, Ansatzpunkte und Maßnahmen identifiziert, die hier nur verkürzt und allgemein dargestellt werden konnten [4]. Es bestehen noch vielfältige Unsicherheiten, die auf einen klaren Forschungsbedarf hinweisen. Naturgemäß gibt es stark kontroverse Einschätzungen hinsichtlich der makro- und sozio-ökonomischen Wirkungen und infolgedessen ihrer politischen Umsetzbarkeit. Die Ressourceneffekte der betrachteten Instrumente sind natürlich stark abhängig von den Annahmen zur konkreten Umsetzung, speziell bei marktbasierten Ansätzen. In der Literatur finden sich jedoch zahlreiche Hinweise auf positive soziale Nebeneffekte, die nicht unmittelbar als Ressourceneffekte angesehen werden können und einer differenzierteren Analyse bedürfen.

Die Folgeeffekte einer gestärkten Regionalwirtschaft für Einkommen, Beschäftigung und kommunale Haushalte sowie die Auswirkungen auf das Innovationsverhalten und Investitionen von Open Sources sind aus heutiger Sicht noch weitgehend unklar. Empirische Kontroversen bestehen in den Bereichen Arbeitszeitverkürzungen (Sinken der Arbeitslosenquote vs. Beschäftigungsrückgänge), ökologische Steuern (erodierende Steuerbasis, Ausmaß der Regressivität, wenn keine Zweckbindung vorliegt, potenzielle Belastungen der Wirtschaft, schwierige EU-Harmonisierung von Steuern allgemein), Verlängerung von Produktlebensdauern (erforderliche Investitionen vs. Entwertung des bestehenden Know-hows), ökologisches Grundeinkommen (Wirkungen auf die individuelle Arbeitsmotivation, unentgeltliche Eigen- und Sorgearbeit im Zielkonflikt mit der sozialpolitischen Leitidee, mehr Teilhabe durch (Erwerbs)-Beschäftigung). Die wachstumshemmende Wirkung von Werbebeschränkungen oder -verboten dürfte derzeit noch sehr klar im Zielkonflikt mit der Ausrichtung von Wirtschaftsinteressen stehen.

Die Frage „Umsetzbarkeit“ ist die Schlüsselherausforderung. Schon in den hier quasi ausschnittartig untersuchten Instrumentenbereichen zeichnet sich ab, dass es eindeutige und gestaltungsmächtige Gegeninteressen gibt. Ein umfassender Politikmix ist mit hohen Anforderungen an Kohärenz, Konsistenz und Glaubwürdigkeit konfrontiert, wobei sich für Deutschland zur Erhöhung von Richtungssicherheit und Umsetzungsfähigkeit die weitergehende Prüfung und Erforschung der teils sehr fortschrittlichen und innovativen Umsetzungsbeispiele aus anderen Ländern empfiehlt.

\section{Anmerkungen}

[1] Siehe Ökologisches Wirtschaften, Vorsorge und Postwachstum, Nr. 1 (2019). An dieser Teilstudie waren neben den Autoren B. Best, J. Benke, J. Buhl, L. Galinski, R. Lucas, C. Koop sowie mit Beiträgen und Kommentaren N. aus dem Moore, T. Korfhage, S. Werland, H. Berg, D. Eichhorn, J. Günther beteiligt.

[2] So zum Beispiel diverse fiskalische, marktbasierte, produktbezogene und regionale Ansätze.

[3] Dazu wurden jeweils Experteneinschätzungen vorgenommen, die in einem iterativen Prozess validiert wurden (Erstautor(en), Gegenlesende, 1. Review, 2. Review, Überarbeitung) und zudem Gegenstand der Diskussion in einem Fachgespräch mit weiteren externen Expert/innen waren.

[4] Die umfangreichen Einzelheiten sind dem Projektendbericht zu entnehmen, der in Kürze als UBA-Text veröffentlicht wird.

\section{Literatur}

Bachus, K./Vanswijgenhoven, F. (2017): The Use of regulatory Taxation as a Policy Instrument for Sustainability Transitions: Old Wine in New Bottles or Unexplored Potential? In: Journal of Environmental Planning and Management 61/9: 1469-1486.

Bahn-Walkowiak, B./Wilts, H./Meyer, M./Distelkamp, M. (2017): Conceptualising a Tax Policy Mix for Resource Efficiency. Selected Results from a Three Transition Pathways Approach. In: Weishaar, S. E./Kreiser, L./Milne, J. E./Ashiabor, H./Mehling, M. (Hrsg.): The Green Market Transition. Carbon Taxes, Energy Subsidies and Smart Instrument Mixes. Northampton, Edward Elgar. 174-192.

Broehl-Kerner, H./Elander, M./Koch, M./Vendramin, C. (2012): Second Life. Wiederverwendung gebrauchter Elektro- und Elektronikgeräte. DessauRoßlau, Umweltbundesamt.

Buhl, J./Acosta Fernandez, J. (2016): Labour Markets: Time and Income Effects for Reducing Working Hours in Germany. In: Santarius, T./ Walnum, H. J./Aall, C. (Hrsg.): Rethinking Climate and Energy Policies. New Perspectives on the Rebound Phenomenon. New York, Springer International. 163-181.

Cooke, P. (1997): Regions in a global market: The experience of Wales and Baden-Württemberg. In: Review of International Political Economy 4/2: 349-381.

Deutsches Institut für Wirtschaftsforschung Econ (DIW Econ) (Hrsg.) (2016): Die ökonomische Bedeutung von Werbung.

Dye, R. F./England, R. W. (2010): Assessing the Theory and Practice of Land Value Taxation. Cambridge, Lincoln Institute of Land Policy.

Haywood, L. (2014): Bedingungsloses Grundeinkommen. Eine ökonomische Perspektive. DIW Roundup - Politik im Fokus 33. www.diw. de/documents/publikationen/73/diw_01.c.479933.de/diw_roundup_33_ de.pdf

Henger, R./Schäfer, T. (2015): Mehr Boden für die Grundsteuer - Eine Simulationsanalyse verschiedener Grundsteuermodelle. Köln, Institut der Deutschen Wirtschaft Köln.

Henriksen, L. (2012): Comprehensive Tobacco Marketing Restrictions: Promotion, Packaging, Price and Place. In: Tobaccocontrol 21: 147-153.

Kerkhof, A./Münster, J. (2015): Quantity Restrictions on Advertising, Commercial Media Bias, and Welfare. In: Journal of Public Economics 131: 124-141.

Knight, K./Rosa, E./Schor, J. (2013): Could Working Less Reduce Pressures on the Environment? A Cross-National Panel Analysis of OECD Countries. 1970-2007. In: Global Environmental Change 23: 691-700.

Kornhardt, U. (2009): Ermäßigter Mehrwertsteuersatz für arbeitsintensive Dienstleistungen - Auswirkungen auf das Handwerk. Göttingen, Universität Göttingen.

Ludewig, D. (2017): Wie eine Ökologische Finanzreform die Postwachstumsökonomie fördern kann. In: Adler, F./Schachtschneider, U. (Hrsg.): Postwachstumspolitiken. Wege zur Wachstumsunabhängigen Gesellschaft. München, oekom. 225-237.

Maitra-Ekern, E./Dalhammar, C. (2016): Regulating Planned Obsolescence: A Review of Legal Approaches to Increase Product Durability and Repar- 
ability in Europe. In: Review of European, Comparative \& International Environmental Law 25/3: 378-394.

Meyer, M. (2015): Simulationsstudien der Potenziale von Gütersteuern zur Reduktion der internationalen Ressourceninanspruchnahme. Berlin, Forschungszentrum für Umweltpolitik.

Meyer, M./Hirschnitz-Garbers, M./Distelkamp, M. (2018): Contemporary Resource Policy and Decoupling Trends. Lessons Learnt from Integrated Model-Based Assessments. In: Sustainability 10/6: 1858-1886.

Petschow, U./Ferdinand, J. P./Dickel, S./Flämig, H./Steinfeldt, M./Worobei, A. (2014): Dezentrale Produktion, 3-D-Druck und Nachhaltigkeit. Berlin, Institut für ökologische Wirtschaftsforschung.

Petschow, U. et al. (2019): Gesellschaftliches Wohlergehen innerhalb der planetaren Grenzen. Die vorsorgeorientierte Postwachstumsposition. In: Ökologisches Wirtschaften 34/1: 15-16.

Place, C./Bindewald, L. (2015): Validating and improving the impact of complementary currency systems through impact assessment frameworks. Int. J. Community Curr. Res. 19, D: 152-164.

Poppe, E. (2014): Reparaturpolitik in Deutschland. Zwischen Produktverschleiß und Ersatzteilnot. Berlin, Sustainum Institut für zukunftsfähiges Wirtschaften.

Santarius, T. (2015): Der Rebound-Effekt. Ökonomische, psychische und soziale Herausforderungen für die Entkopplung von Wirtschaftswachstum und Energieverbrauch. Marburg, Metropolis.

Schachtschneider, U. (2014): Freiheit, Gleichheit, Gelassenheit. Mit dem Ökologischen Grundeinkommen aus der Wachstumsfalle. München, oekom.

Schiller, G./Deilmann, C. (2010): Ermittlung von Ressourcenschonungspotenzialen bei der Verwertung von Bauabfällen und Erarbeitung von Empfehlungen zu deren Nutzung. Dessau-Roßlau: Umweltbundesamt.

Stadt Zürich (Hrsg.) (2009): Ressourcenstrategie „Bauwerk der Stadt Zürich“. Materialflüsse und Energiebedarf bis 2050. Zürich, Amt für Hochbauten.
Townsend, A./Jeffery, L./Fidler, D./Crawford, M. (2011): The Future of Open Fabrication. Report. Palo Alto, Institute of the Future. Palo Alto.

Umweltbundesamt (Hrsg.) (2016): Einfluss der Nutzungsdauer von Produkten auf ihre Umweltwirkung. Schaffung einer Informationsgrundlage und Entwicklung von Strategien gegen "Obsoleszenz".

Umweltbundesamt (2018): Umweltbezogene Steuern und Gebühren. www.umweltbundesamt.de/daten/umwelt-wirtschaft/umweltbezogenesteuern-gebuehren\#textpart-1

Umweltbundesamt (i. E.): Ansätze zur Ressourcenschonung im Kontext von Postwachstumskonzepten. Endbericht des Projekts. Forschungskennzahl 3715311040 .

\section{AUTOR/INNEN + KONTAKT}

Bettina Bahn-Walkowiak ist Projektleiterin in der Abteilung Kreislaufwirtschaft am Wuppertal Institut für Klima, Umwelt, Energie.

Dr. Henning Wilts ist Abteilungsleiter der Abteilung Kreislaufwirtschaft am Wuppertal Institut für Klima, Umwelt, Energie.

Wuppertal Institut für Klima, Umwelt, Energie $\mathrm{GmbH}$, gemeinnützig, Döppersberg 19, 42103 Wuppertal. Tel.: +49 202 24920, E-Mail: bettina.bahn-walkowiak@wupperinst.org, henning.wilts@wupperinst.org, Internet: https://wupperinst.org/
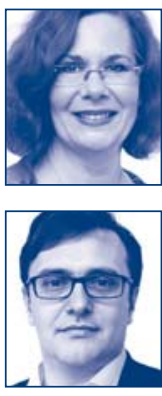